
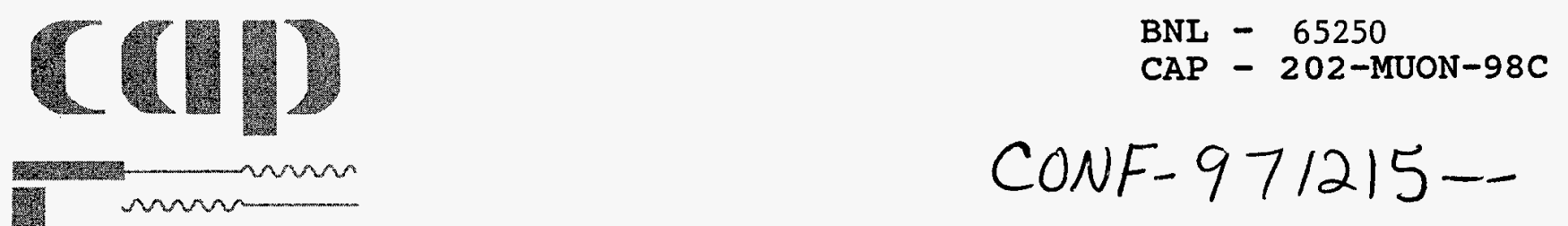

IONIZATION COOLING AND MUON DYNAMICS*

Zohreh Parsa

Department of Physics

Brookhaven National Laboratory

Upton, NY 11973

RECEIVED

APR 081988

OSTI

*This work was performed under the auspices of the U.S. Department of Energy under Contract No. DE-ACO2-76CHOOO16.

DISTRIBUTION OF THIS DOCUMENT IS UNLIMTTED

January 1998

MASTER

19980504059

CENTER FOR ACCELERATOR PHYSICS

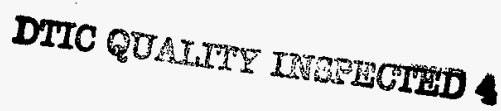

BROOKHAVEN NATIONAL LABORATORY

ASSOCIATED UNIVERSITIES, INC.

Under Contract No. DE-AC02-76CH00016 with the

UNITED STATES DEPARTMENT OF ENERGY

Submitred to "4th Int'l. Conference on "Physics Potential \& Development of Muon-Muon Colliders", San Francisco, CA, Dec. 10-12, 1997. 


\section{DISCLAIMER}

This report was prepared as an account of work sponsored by an agency of the United States Government. Neither the United States Government nor any agency thereof, nor any of their employees, nor any of their contractors, subcontractors, or their employees, makes any warranty, express or implied, or assumes any legal liability or responsibility for the accuracy, completeness, or usefulness of any information, apparatus, product, or process disclosed, or represents that its use would not infringe privately owned rights. Reference herein to any specific commercial product, process, or service by trade name, trademark, manufacturer, or otherwise, does not necessarily constitute or imply its endorsement, recommendation, or fa voring by the United States Government or any agency, contractor or subcontractor thereof. The views and opinions of authors expressed herein do not necessarily state or reflect those of the United States Government or any agency; contractor or subcontractor thereof. 


\title{
Ionization Cooling and Muon Dynamics
}

\author{
Zohreh Parsa \\ Brookhaven National Laboratory ${ }^{1}$ \\ Physics Department, $901 \mathrm{~A}$ \\ Upton, New York 11973-5000, USA. \\ e-mail: parsa@bnl.gov
}

\begin{abstract}
Muon colliders potential to provide a probe for fundamental particle physics is very interesting. To. obtain the needed collider luminosity, the phase-space volume must be greatly reduced within the muon life time. The Ionization cooling is the preferred method used to compress the phase space and reduce the emittance to obtain high luminosity muon beams. We note that, the ionization losses results not only in damping, but also heating. We discuss methods used including moments methods, Focker-Plank Equation, and Multi Particle Codes. In addition we show how a simple analysis permits us to estimate the most part of the optimal system parameters, such as optimal damping rates, length of the system and energy.
\end{abstract}

\section{INTRODUCTION}

To obtaine very intense muon beams needed for a high-luminosity muon collider a high intensity proton source is bunch compressed and focused on a heavy metal target. The pions generated are captured and transferred to a decay channel. Since the muons are created through pion decay into a diffuse phase space, some form of cooling is necessary, to compress the phase space and reduce the emittance. Ionization is the preferred method, where the muons passing through a material medium lose momentum and energy through ionization interactions in transverse and longitudinal directions. The normalized emittance is reduced due to transvers energy losses. Although Ionization cooling studies dates back to 1970 (see e.g. [1-5]), experimentally it is a new technique that has not yet been demonstrated. We discuss the formalism including equations for damping rates of particles, optimization criterion, etc. We show how to estimate the optimal damping, energy and length of the system and illustrate the use of our formulations, with a numerical example.

1) Supported by US Department of Energy Contract No. DE-AC02-76CH00016. 


\section{FORMALISM}

At present time principles of the ionization cooling are well known and established. However, some details of the theory are not completely clear. A milestone of the theory are equations for damping rates of an individual particle. If longitudinal ionization cooling is absent transverse damping rates are defined by the following equation:

$$
\lambda_{x}(z)=\lambda_{y}(z)=\frac{\frac{d E^{\text {ion }}}{d z}(z)}{m c^{2} \beta^{2}(z) \gamma(z)}=\lambda_{\perp}(z)
$$

Here $x, y$ are transverse variables and $z$ is a longitudinal variable.

$\frac{d E^{\text {ion }}}{d z}(z)=$ ionization energy losses; $m$ is the muon mass; $\beta, \gamma$ are the relativistic muon parameters. Where $\lambda_{\perp}$ and $\lambda_{\|}$are natural transverse and longitudinal damping rates (decrements) respectively.

Longitudinal ionization cooling may be introduced artificially, if: 1) we use section with dependence $\left.\frac{d E^{\text {ion }}}{d z}(x, z) ; 2\right)$ this section is designed in such a way as to have dispersion function $\Psi_{x}$ inside. Then

$$
\lambda_{\|}(z)=\lambda_{\perp}(z) \frac{\Psi_{x}(z)}{L(z)}
$$

Here

$$
L(z)=\frac{\left(\frac{d E}{d z}\right)^{\text {ion }}(0, z)}{\frac{d}{d x}\left(\frac{d E}{d z}\right)^{\text {ion }}(0, z)}
$$

However, introduction of $\lambda_{\|}(z)$ changes a corresponding damping rate $\lambda_{x}(z)[2,9]$

$$
\lambda_{x}(z)=\lambda_{\perp}(z)-\lambda_{\|}(z)
$$

In general case, there is a fundamental theorem [2], that

$$
\sum_{i} \lambda_{i}(z)=2 \lambda_{\perp}(z)
$$

Thus, we see, that we can only redistribute the damping rates.

However, each section with ionization losses give not only damping, but a heating as well: transverse heating appears due to multiple Coulomb scattering and longitudinal one is due to so named "straggling" of the ionization losses (we note that, this straggling is produced by fast "knock-on" ionization electrons).

From theoretical point of view, a situation with ionization cooling completely corresponds to a situation with radiation cooling whose theory is well developed. 
There is some standard "hierarchy" of methods for analyzing such systems: i) Moments methods, ii) Focker-Planck equation, iii) Multi-particles codes.

i) Method of moments (MM)

Using equation of motion for separate particles or kinetic equation, it is easy to derive equations for the second order moments $\left(\left\langle x^{2}\right\rangle,\left\langle x x^{\prime}\right\rangle,\left\langle x^{\prime 2}\right\rangle\right.$ and etc.). (see, for example, [11]). Analysis of these equations permits us to find equilibrium emittances and to investigate evolution of emittances with time for linear external fields. It is necessary to be very careful when using this method (MM) e.g., 1) In transverse plane a strong focusing usually is used, and therefore "smoothing" of the motion (change $w_{\perp}^{2}(z) \rightarrow w_{0}^{2}$ ) must be done very accurately. 2) Often some authors have solved only the equations for $\left\langle x^{2}\right\rangle$ (which, really, is proportional to beam temperature) without solving the equations for the last moments. However, to calculate evolution on time accurately we must solve the system of coupled equations for the moments $[6,7,11]$.

ii) Focker-Planck (FP) equation

To find a form of a distribution function and to estimate beam losses due to diffusion it is possible to use the FP equation. The one-dimensional FP equation has the following form:

$$
D \frac{\partial}{\partial I}\left(I \frac{\partial f}{\partial I}\right)+\alpha \frac{\partial}{\partial I}(f I)=\frac{\partial f}{\partial z}
$$

Where $f=$ the distribution function $(f=f(I, z)) ; I=$ action; $z=$ longitudinal variable; $D=$ the diffusion coefficient; and $\alpha=$ the damping coefficient. The FP equation must be solved using the following boundary condition:

$$
f_{m}\left(I_{m}, z\right)=0
$$

where $I_{m}$ is the aperture in $I$-variable. If $I_{m}=\infty$, Eq. (5) has a stationary solution:

$$
f_{0}=\frac{1}{I_{o}} \exp \left(-\frac{I}{I_{0}}\right)
$$

Where $I_{0}=\frac{D}{\alpha}$.

Let us underline, that if $I_{m}>>I_{0}$, we can estimate particle losses using a simple formula:

$$
\begin{gathered}
\frac{1}{N} \frac{d N}{d z} \simeq D \frac{I_{m} \partial f_{0}}{\partial I}\left(I=I_{m}\right)=\frac{D I_{m}}{I_{0}^{2}} \exp \left(-\frac{I_{m}}{I_{0}}\right)= \\
\alpha \frac{I_{m}}{I_{0}} \exp \left(-\frac{I_{m}}{I_{0}}\right)
\end{gathered}
$$

If $\frac{I_{m}}{I_{0}} \simeq$ constant

$$
\frac{\Delta N}{N} \sim \alpha L \frac{I_{m}}{I_{0}} \exp \left(-\frac{I_{m}}{I_{0}}\right)
$$


For example, if $\frac{I_{m}}{I_{0}}=9$ and $L=\frac{5}{\alpha}$ (that corresponds to damping of an initial emittance in 150 times), three-dimensional diffusion losses $\frac{\Delta N}{N} \sim 0.017$

If condition $I_{m}>>I_{0}$ is not performed, we can find a beam evolution by numerical solution of FP equation.

iii) Multi-particle codes.

We see that method of moments (MM) and Focker-Planck (FP) equation gives only limited information. A whole information about the beam, including largeangle scattering, interactions with residual gas, increase of emittances due to nonlinearity of the external field and so on can be found only by multi-particle codes. Map methods are not convenient for our problem due to presence of large noises. However, we note, that it is a correct procedure that can be used to validate accuracy of the final results of numerical calculations.

\section{OPTIMIZATION AND CALCULATION OF PARAMETERS}

Problem of choice of optimal parameters for the muon cooling system is open, and its solution is far from trivial due to infinite numbers of possible options. The process of optimization can usually be divided into five stages: 1) choice of optimization criterion; 2) calculation of "primary" parameters by use of simple analytical models; 3 ) choice of design of focusing and accelerating systems; 4) search of optimum for chosen focusing and accelerating systems by use of more sophisticated algorithms; 5) validation of chosen scheme by use of multi-particles codes, etc. Here we discuss the first two points (others are not of scope of this paper).

- Optimization: Luminosity of collider $L$ is defined by the following expression:

$$
L \sim \frac{N^{2} f}{g_{x} g_{y}}=\frac{N^{2} f}{\epsilon_{\perp}^{f} \cdot \beta_{\perp}^{f}}
$$

Where $N=$ a number of muons per bunch, $f=$ mean repetition frequency of collisions, $\epsilon_{\perp}^{f}=$ emittance at collision point and $\beta_{\perp}^{f}=\beta$-function at collision point. Usually $\beta_{\perp}^{f}$ is limited by condition:

$$
\beta_{\perp}^{f} \geq \sigma_{z}^{f}
$$

where $\sigma_{z}^{f}$ is a longitudinal bunch size. Let us assume, that: 1) $\frac{\Delta p_{f}}{p}$ is known (monochromatic experiments); 2) we can redistribute emittances inside a given sixdimensional phase volume. Then, taking into account losses in the cooling system, we can rewrite Eq. (11) in the following form:

$$
L \sim \frac{N_{0}^{2} \exp \left(-\frac{2}{c T_{0}} \int_{0}^{z} \frac{d z}{\gamma(z)}\right) D^{2}}{\sqrt{V_{6}^{N} \cdot \epsilon_{\|}^{f}}} \cdot\left(\frac{\Delta p}{p}\right)_{\|}^{f}
$$


Here " $N_{0}$ " is a number of particles at an entrance of the cooling system, "exp" describes muon decay, " $D$ " describes muon losses in cooling section, and " $V_{6}^{N}$ " is an invariant six-dimensional phase volume of muon beam.

Thus we can introduce "merit factor" which describes a quality of muon cooling system. We obtain

$$
R=\frac{D^{2} \exp \left[-\frac{2}{c T_{0}} \int_{0}^{z} \frac{d_{z}}{\gamma(z)}\right]}{\sqrt{V_{6}^{N}}}
$$

Note that, the dependence on $V_{6}^{N}$ may be stronger. With account of all the circumstances, we can write

$$
R \sim\left(V_{6}^{N}\right)^{\alpha}
$$

with $\alpha$ in interval $(0.5 ; 2 / 3)$.

- Calculation of primary parameters

Let us neglect muon losses $\left(D=1, \exp \left[-\frac{2}{c T_{0}} \int_{0}^{z} \frac{d z}{\gamma(z)}\right]=1\right)$. Then to maximize $R$ we must minimize $V_{6}^{N}$. Taking into account standard formula for equilibrium phase volumes, we have:

$$
V_{6}^{N}=\frac{W_{\perp}^{2} \cdot W_{\|}}{\alpha_{\|}\left(\alpha_{\perp}^{0}-\frac{\alpha_{\|}}{2}\right)^{2}}
$$

This equation has a solution relative to $\alpha_{\|}$:

$$
\begin{aligned}
\alpha_{\perp}^{\min }=\alpha_{\|}^{\min } & =\frac{2 \alpha_{\perp}^{0}}{3} ; \\
\left(\sqrt{V_{6}^{N}}\right)_{\min } & =\frac{27}{8} \frac{W_{\perp}^{2} \cdot W_{\|}}{\left(\alpha_{\perp}^{0}\right)^{3}}
\end{aligned}
$$

However, a situation will change if we take into account the muon losses.

As an example, let us consider a simplest case: 1) $D=1$; 2) $\lambda_{\|}<<\Omega$; 3) $\gamma=\gamma_{0}$. In this case (for $\left.\lambda_{\perp}=\lambda_{\|}\right)$

$$
R \simeq \frac{\exp \left[-2 L / c T_{0} \gamma_{0}\right]}{\left(V_{6}^{N}\right)_{\min }^{\frac{1}{2}}\left[1+\frac{\epsilon_{\perp}^{0}-\epsilon_{e q}}{\epsilon_{e q}} \exp (-\lambda L)\right]^{-3 / 2}}
$$

It is easy to see that in this case there is an optimal system length $L$, which is defined by the following equation:

$$
L_{0}=\frac{1}{\lambda} \ln \left(\frac{\frac{A B}{\lambda}}{\frac{3}{2} B-\frac{A}{\lambda}}\right)^{-1}
$$


Where $A=\frac{2}{c T_{0} \gamma_{0}}$ and $B=\frac{\epsilon_{\perp}^{0}-\epsilon_{e q}}{\epsilon_{e q}}$.

Numerical Example: We consider a numerical example with $\gamma_{0}=1.5 ; \lambda=5$. $10^{-2} \mathrm{~m}^{-1} ; B=10$. Then we obtain:

$$
\begin{aligned}
\tau_{0}^{\max } & =\lambda L=3.6 \\
R_{\max } & \simeq \frac{0.6}{\sqrt{\left(\sqrt{V_{6}^{N}}\right)_{\min }}}
\end{aligned}
$$

\section{DISCUSSION}

We discussed the formulation and methods used to study the muon dynamics. We did not try to present all the questions in detail. We illustrated and would like to underline, that simple analysis permits us to estimate the most part of the optimal system parameters, such as optimal damping rates, length of the system, energy, etc.

\section{REFERENCES}

1. A. Skrinsky, Colliding Beams Program in Novosibirsk, International Seminar on Prospects in High Energy Physics; Morges, 1971.

2. V. Parchochuk and A. Skrinsky, Methods of cooling of charged particle beams, Phys. of Elementary Part. and Atomic Nucl., 12 557-613 (1981).

3. Physics Potential and Development of $\mu^{+} \mu^{-}$Colliders Workshop - AIP Conf. Proceedings, 352, AIP Press, Woodbury, NY.

4. The 9th Advanced ICFA Beams Dynamics Workshop - AIP Conf. Proceedings, 372.

5. $\mu^{+} \mu^{-}$Collider. A Feasibility Study, BNL-52503.

6. T. Vserolozhskaya, Kinetics of ionization cooling of muons, AIP CP 372, p. 159 , (1996).

7. Z. Parsa and P. Zenkevich, Kinetics of Muon Longitudinal cooling in Proceedings of the 1996 Beam Stability and Nonlinear Dynamics Symposia AIP CP 405, p.165-172.

8. Z. Parsa and P. Zenkevich, unpublished, ITP-96.

9. D. Neuffer, Muon cooling and applications, Montreux Conference on Beam Cooling, Proc., p. 49, 1993.

10. Z. Parsa and P. Zenkevich, Application of Moments Method to Dynamics of Muon Cooling System in Beam Stability and Nonlinear Dynamics AIP CP 405, p.183-188, 1997.

11. Z. Parsa, Emittance and Moments in Accelerators and Beamlines Parts (I) and (II), Reports and BNL-44618, (10/1989); W. Lysenko, Z. Parsa, in Beam Stability and Nonlinear Dynamics AIP CP 405, p. 211-222, 1997. 


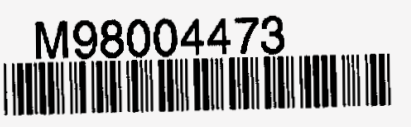

Report Number (14) BNL-- 65250

$\frac{\text { CONF-971215- }}{\text { CAP--202-M4ON-98C }}$

Publ. Date (11)

Sponsor Code (18)

UC Category (19)

$\frac{\frac{199801}{D O E / E R}, \text { XF }}{\text { UC-414, DOE/ER }}$

